\title{
Radiation-induced optic neuropathy following external beam radiation therapy for nasopharyngeal carcinoma: A retrospective case-control study
}

\author{
WEI WANG $^{1 *}$, HUI YANG ${ }^{1 *}$, LING GUO $^{2}$, HONGYU SU $^{1}$, SHIHUI WEI $^{3}$ and XIULAN ZHANG ${ }^{1}$ \\ ${ }^{1}$ Zhongshan Ophthalmic Center, State Key Laboratory of Ophthalmology; ${ }^{2}$ Sun Yat-sen University Cancer Center \\ State Key Laboratory of Oncology in South China, Sun Yat-sen University, Guangzhou, Guangdong 510060; \\ ${ }^{3}$ Department of Ophthalmology, General Hospital of the People's Liberation Army, Beijing 100000, P.R. China
}

Received November 26, 2014; Accepted February 6, 2015

DOI: $10.3892 / \mathrm{mco} .2016 .787$

\begin{abstract}
Radiation-induced optic neuropathy (RION) is a severe ocular complication in patients with nasopharyngeal carcinoma (NPC) following external beam radiation therapy. However, the systemic risk factors for this condition remain unclear. Therefore, patients with NPC who received radiotherapy between 2004 and 2007 at the Sun Yat-Sen University Cancer Center were retrospectively reviewed in this case-control study. The study included 40 RION patients and 40 patients in the control group, who were strictly matched to the RION patients by tumor histopathology, location, Union for International Cancer Control-Tumor Node Metastasis classification and radiotherapy protocol. Univariate and multivariate statistical regression analyses were performed to identify factors predictive of RION. The univariate analysis demonstrated that age ( $>60$ years), gender (female) and chemotherapy significantly affected the risk of RION, whereas diabetes, hypertension and hepatitis B virus infection did not exert a significant effect. The results of the multivariate analysis suggested that only gender and chemotherapy were significantly associated with an increased incidence of RION. Therefore, the results of the present study suggested that female gender and chemotherapy constitute risk factors for the development of RION following radiotherapy for NPC. The ocular symptoms of high-risk patients should be carefully investigated and reported by ophthalmologists.
\end{abstract}

Correspondence to: Professor Xiulan Zhang, Zhongshan Ophthalmic Center, State Key Laboratory of Ophthalmology, Sun Yat-sen University, 54S Xianlie Road, Guangzhou, Guangdong 510060, P.R. China

E-mail: zhangx12@mail.sysu.edu.cn

*Contributed equally

Key words: radiation-induced optic neuropathy, risk factor, nasopharyngeal carcinoma

\section{Introduction}

Nasopharyngeal carcinoma (NPC) is a major health concern worldwide. According to the cancer statistics, there were an estimated 84,400 cases of NPC and 51,600 deaths in 2008, representing $\sim 0.7 \%$ of the global cancer burden (1). NPC is endemic in Southeast Asia, particularly in Southern China. An endemic pocket is observed in Guangdong Province, with a high incidence of 20-30/100,000 person-years, which is the highest worldwide (2). NPC treatment usually involves radiation therapy, chemotherapy or a combination of the two modalities. Radiotherapy remains the primary and occasionally the only effective treatment for NPC due to its high rate of response (3).

Radiation-induced optic neuropathy (RION) is one of the most common complications that may occur in patients treated with radiotherapy for NPC (4). RION usually presents with acute, profound, irreversible visual loss in one or both eyes after a latency of months to years following radiation exposure. RION is considered to be a result of radiation necrosis of the anterior visual pathway. Despite its rarity, the disabling damage to vision severely affects the patients' quality of life, with the most severely affected eyes exhibiting a final visual acuity of $<20 / 200$, whereas several cases progress to complete blindness (5). RION has long presented radiologists and ophthalmologists with a challenge. With the advances in radiation modalities, NPC patients achieve prolonged survival. Therefore, the quality of life of the survivors requires more attention, despite the rarity of RION.

Although RION has been documented for $\sim 100$ years, the treatment results in reported studies are disappointing. The visual function progressively deteriorates once visual loss has emerged. Thus, identifying the predictive factors for developing RION may modify follow-up for patients at risk, which may enable earlier management of the developing radiation-induced optic changes. Several studies have been performed to investigate the radiation-associated factors, including dosage to the optic nerve and fraction size, which are known as significant risk factors for the development of RION (6-9). However, other concurrent factors, including chemotherapy and other comorbidities, such as diabetes 
mellitus, have been less extensively investigated and the results are controversial $(7,8)$. In this study, we performed a tumor classification- and treatment-matched case-control study to investigate the association between RION and systemic risk factors in NPC patients who receive radiotherapy.

\section{Materials and methods}

Patients. Between 2004 and 2007, a total of 4,508 NPC patients were treated with external beam radiation therapy at Sun Yat-sen University Cancer Center, Guangzhou, Guangdong, China. A retrospective electronic chart review was conducted. The patients were followed up until December, 2013. Research Ethics Board approval for retrospective data collection was obtained. The research followed the tenets of the Declaration of Helsinki and written informed consent was obtained from all the participants prior to enrollment. The patients were recalled and evaluated by telephone interview. All the patients received radiotherapy for the first time. Among these, 40 patients who developed RION following radiotherapy were identified and confirmed by retrospective review of the medical records of Sun Yat-sen University Cancer Center and Zhongshan Ophthalmic Center, or by thorough ophthalmic tests between October, 2012 and December, 2013. The RION patients had no known retinopathy, neuropathy, glaucoma, cataract, keratitis sequelae, or vasculopathy due to diabetes or hypertension prior to radiotherapy and did not present with severe loss of vision as a result of RION, with a Karnofsky performance status score of $\geq 70$.

Of the 4,508 NPC patients, 40 who were strictly matched with the RION patients by tumor histopathology, location, Union for International Cancer Control (UICC)-Tumor Node Metastasis classification and radiotherapy, were included in the control group. The exclusion criteria were as follows: Any advanced refractive error or media opacity impairing vision (keratitis sequelae, cataract, vitreal pathological characteristics), retinopathy, glaucoma, optic neuropathy, or vasculopathy due to diabetes or hypertension.

Radiotherapy. All the patients were diagnosed with NPC by nasoendoscopy and biopsy. No locoregional recurrence or distant metastases were observed. NPC was staged according to the 6th edition of the UICC staging system, 2002. The patients in the case and control groups were treated with identical external beam radiation protocols, using conventional linear accelerators with $8 \mathrm{MV}$ photons or ${ }^{60} \mathrm{Co}$ unit, in fractions of 2 Gy/day, 5 days/week, through opposed lateral fields covering the primary tumor and the upper cervical lymphatics. On the nasopharyngeal primary site and neck, the field of radiotherapy was set up according to the state standard of China for diagnosis and treatment of cancer and the stage of the NPC. The pre-auditory fields were conventionally used as the main field of radiotherapy. The field of radiotherapy was restricted to the nasopharyngeal primary site by a cerrobend. Anterior and posterior tangential fields and bilateral vertical fields at the neck were used. However, the field of radiotherapy unavoidably involved a part of the optic nerve, optic chiasma, brainstem and other important organs in the head and neck region. The radiotherapy dose was symmetric to the right and left sides. The total dose was 60-80 Gy to the nasopharynx and 70 Gy to the neck for all the subects. The total treatment duration was 49-56 days.
Diagnostic criteria of RION. The diagnosis of RION was established during the regular follow-up visits or through examinations due to vision changes according to the following criteria $(4,10)$ : i) History of radiotherapy; ii) painless progressive visual loss; iii) visual field defect and abnormal visual evoked potential; and/or iv) ophthalmoscopic evidence of blurring of the optic disc margins, dilation of retinal veins, hemorrhages, exudates and neovascularization. Additional findings included microvascular incompetence, evidenced by areas of capillary non-perfusion of the disc and nearby neural retina in fluorescein angiography. The excluding criteria were as follows: i) Tumor recurrence or metastasis; ii) nerve damage following radiation for cerebral arachnoiditis; and iii) other diseases which may lead to visual loss, such as acute ischemic optic neuropathy or giant cell arteritis.

Statistical analysis. Descriptive statistics were used to summarize the basic characteristics of the patients included in the case and control groups. Normally distributed data were expressed as mean \pm standard deviation. An independent samples t-test was used to compare the two groups. If the data exhibited a non-parametric distribution, the Mann-Whitney U test was used. The differences between categorical covariates were evaluated by the $\chi^{2}$ test. The association between RION and other variables was analyzed by the logistic regression method. All the factors were entered in a univariate analysis and those with significant difference $(\mathrm{P}<0.1)$ were selected for the multivariate analysis. $\mathrm{P} \leq 0.05$ was considered to indicate statistically significant differences.

\section{Results}

Patient characteristics. The demographics and medical history of the patients are summarized in Table I. All the patients had priorly received radiotherapy for NPC. There were no selection criteria regarding patient gender, age, or eye laterality. The case group involved 40 patients who developed RION and matched our enrollment criteria. A total of 12 patients $(30 \%)$ received concurrent chemotherapy and 23 received radiotherapy alone. The case group included 9 men and the mean patient age was $56.6 \pm 9.4$ years. In total, 4 patients $(10 \%)$ had concurrent diabetes and $2(5 \%)$ had hypertension. The patients in the control group were matched to the case group by tumor type, stage, histology and radiotherapy protocol.

Univariate and multivariate analysis. Following adjustment for dosage, tumor stage, subtype, location and radiotherapeutic modality, the associated factors were analysed by logistic regression. In the univariate analysis, age $(\mathrm{P}=0.012)$, gender $(\mathrm{P}=0.006)$ and chemotherapy $(\mathrm{P}<0.001)$ were significantly associated with the risk of developing RION (Table II), whereas there was no significant association with comordities, such as diabetes melitus, hypertension, or HBV infection. In the multivariate analysis, only female gender and chemotherapy were statistically significantly associated with the risk of RION (Table III).

\section{Discussion}

RION is a devastating late complication affecting the anterior visual pathway in NPC patients following radiotherapy. 
Table I. Demographic characteristics of the cases and controls.

\begin{tabular}{|c|c|c|c|}
\hline Characteristics & Cases $(n=40)$ & Controls $(n=40)$ & P-value \\
\hline Age, years $($ mean $\pm S D)$ & $56.6 \pm 9.4$ & $52.6 \pm 12.8$ & 0.512 \\
\hline Gender (male/female) & $9 / 31$ & $3 / 37$ & 0.115 \\
\hline Histopathology, no. (\%) & & & NA \\
\hline Undifferentiated non-keratinizing carcinoma & $29(72.5)$ & $29(73.0)$ & \\
\hline Differentiated non-keratinizing carcinoma & $3(7.5)$ & $3(7.5)$ & \\
\hline Poorly differentiated keratinizing carcinoma & $8(20.0)$ & $8(20.0)$ & \\
\hline Tumor locations, no. (\%) & & & NA \\
\hline Left top wall & $5(12.5)$ & $5(12.5)$ & \\
\hline Right top wall & $7(17.5)$ & $7(17.5)$ & \\
\hline Bilateral top wall & $28(70.0)$ & $28(70.0)$ & \\
\hline UICC TNM classification, no. (\%) & & & NA \\
\hline T0N2M0 & $1(2.5)$ & $1(2.5)$ & \\
\hline T1N0M0 & $1(2.5)$ & $1(2.5)$ & \\
\hline T2N0M0 & $5(12.5)$ & $5(12.5)$ & \\
\hline T3N0M0 & $3(7.5)$ & $3(7.5)$ & \\
\hline T3N0M1 & $1(2.5)$ & $1(2.5)$ & \\
\hline T3N1M0 & $5(12.5)$ & $5(12.5)$ & \\
\hline T3N2M0 & $5(12.5)$ & $5(12.5)$ & \\
\hline T3N0M0 & $2(5.0)$ & $2(5.0)$ & \\
\hline T4N0M0 & $6(15.0)$ & $4(15.0)$ & \\
\hline T4N1M0 & $4(10.0)$ & $4(10.0)$ & \\
\hline T4N2M0 & $5(12.5)$ & $5(12.5)$ & \\
\hline T4N3M0 & $2(5.0)$ & $2(5.0)$ & \\
\hline Chemotherapy (yes/no) & $18 / 22$ & $3 / 37$ & 0.010 \\
\hline Diabetes mellitus (yes/no) & $4 / 36$ & $2 / 38$ & 0.396 \\
\hline Hypertension (yes/no) & $2 / 38$ & $1 / 39$ & 0.556 \\
\hline HBV-positive (yes/no) & $18 / 22$ & $15 / 25$ & 0.500 \\
\hline
\end{tabular}

SD, standard deviation; UICC, Union for International Cancer Control; HBV, hepatitis B virus; NA, not applicable.

Table II. Univariate analysis of demographics and systemic characteristics of patients with nasopharyngeal carcinoma treated with radiotherapy and control subjects.

\begin{tabular}{lccr}
\hline Characteristics & Odds ratio & 95\% confidence interval & P-value \\
\hline Gender (female vs. male) & 1.58 & $1.14-2.19$ & 0.006 \\
Age, years ( $\leq 60$ vs. $>60)$ & 1.48 & $1.09-2.00$ & 0.012 \\
Eye (right vs. left) & 1.44 & $0.55-3.12$ & 0.536 \\
Chemotherapy (yes vs. no) & 1.62 & $1.29-2.05$ & 0.000 \\
Diabetes mellitus (yes vs. no) & 1.2 & $0.78-1.83$ & 0.402 \\
Hypertension (yes vs. no) & 1.18 & $0.66-2.15$ & 0.562 \\
HBV-positive (yes vs. no) & 1.08 & 0.1 .36 & 0.502 \\
\hline
\end{tabular}

HBV, hepatitis B virus.

Table III. Multivariate analysis for the systemic factors predictive of development of radiation-induced optic neuropathy.

\begin{tabular}{lccc}
\hline Predictive factors & Odds ratio & 95\% confidence interval & P-value \\
\hline Gender (female vs. male) & 1.38 & $1.02-1.88$ & 0.039 \\
Chemotherapy (yes vs. no) & 1.47 & $1.15-1.88$ & 0.003 \\
\hline
\end{tabular}


Despite this being a rare complication, the disabling damage to vision severely affects the patients' quality of life and usually results in profound irreversible visual loss. The results of treatment for RION have been disappointing and there is currently no definitive treatment available (5,11-15). Therefore, prevention is crucial. With the advances in radiotherapy techniques, the survival time of NPC patients may be prolonged. Thus, the prevention and management of ocular complications are important for medical practitioners. However, our knowledge on RION is mainly derived from studies investigating the threshold dose to the optic nerve that may cause blindness, rather than identifing other related systemic factors and our understanding of comorbid factors is particularly limited. Therefore, the identification of factors associated with the development of RION is crucial.

We conducted a matched case-control study to investigate risk factors for RION, focusing on controversial factors, such as diabetes and hypertension. The radiation dose to the optic nerve is a well known significant factor for the development of RION. Leber et al (16) investigated patients undergoing radiosurgery and found that the actuarial risk of developing RION was zero for patients receiving a maximum dose of $<10 \mathrm{~Gy}$. By contrast, in patients receiving 10-15 Gy and $>15$ Gy, the risk of RION was 26.7 and $77.8 \%$, respectively. In the present study, we investigated the systemic factors following adjustment for dosage and tumor risk factors. The results of the univariate analysis demonstrated that diabetes and hypertension did not constitute risk factors for the development of RION. However, gender and chemotherapy were found to be significant risk factors after excluding confounding factors.

In a previous study, Bhandare et al (8) observed that 2 patients $(25 \%)$ with hypertension developed RION compared to $22(8 \%)$ who did not have hypertension. Of the 12 patients with diabetes, only $1(8 \%)$ developed RION, compared to $23(9 \%)$ of 261 patients without diabetes. Entezari et al (17) also suggested that arterial hypertension and diabetes are predisposing risk factors for the development of RION. Theoretically, damage to the vascular endothelium and the neuroglial progenitors plays an important role in the development of RION. Systemic factors, such as hypertension and diabetes, may disrupt the circulation around the optic nerve by causing pathological changes in the arterioles. Thus, patients with hypertension and diabetes may be more susceptible to ischemia of the optic nerve. However, diabetes and hypertension were not confirmed as risk factors in this study. As there were only 4 patients with diabetes and 2 with hypertension in our case group, more cases should be included in the study for further investigation.

The use of chemotherapeutic agents may produce drug toxicity-related ocular side effects; these drugs include cis-platinum, vincristine and nitrosoureas (18). Norton et al (19) reported that vincristine may induce neuropathy, including optic neuropathy. The combined use of chemotherapeutic agents and radiation may result in toxicity to the host that is higher compared to that associated with either modality alone. Di Chiro et al (20) hypothesized that the addition of concomitant chemotherapy to radiation therapy increases the risk of RION at lower radiation doses. The concurrent administration of 5-fluorouracil (5-FU) and radiation involving the eye has been used in the treatment of tumors of the nasal cavity and paranasal sinuses. In a clinical study of patients with paranasal sinus malignacies, 22 patients were treated with irradiation alone and 18 were treated with irradiation plus intra-arterial 5-FU. Finally, two-thirds of the patients receiving radiation alone never experienced any visual difficulties; however, significant ocular toxicity was observed in $100 \%$ of patients receiving combination therapy (18). In our study, the combination of chemotherapy with radiotherapy was found to significantly increase the risk of RION, which is consistent with the findings of previous studies.

Age and gender were also considered to be risk factors by previous studies. Demizu et al (7) reported that age was a risk factor and Hasegawa et al (21) reported that gender was a risk factor. Esassolak et al (22) considered RION to be a disease of the middle-aged and elderly patients, although it may affect patients of any age. Our study only identified gender as a risk factor in the multivariate analysis.

The limitations of the present study include the relatively small number of patients assessed and the relatively short follow-up period. In addition, retrospective studies are susceptible to recall bias. However, to the best of our knowledge, this is the first case-control study to identify the systemic factors associated with the development of RION following radiotherapy for NPC. The results of this study identified female gender and chemotherapy as systemic risk factors for RION. A larger prospective study with a longer follow-up period is required to obtain more reliable results. Identifying the predictive factors for developing RION may modify follow-up for patients at risk. Therefore, the ocular symptoms of high-risk patients should be carefully investigated and reported by ophthalmologists.

\section{Acknowledgements}

This study was supported by grants from the Science and Technology Planning Project of Guangdong Province, China (no. 2012B031800353) and the Natural Science Foundation of Guangdong Province, China (no. S2012010008439, Hui Yang).

\section{References}

1. Jemal A, Bray F, Center MM, Ferlay J, Ward E and Forman D: Global cancer statistics. CA Cancer J Clin 61: 69-90, 2011.

2. Cao SM, Simons MJ and Qian CN: The prevalence and prevention of nasopharyngeal carcinoma in China. Chin J Cancer 30: 114-119, 2011.

3. Rottey S, Madani I, Deron P and Van Belle S: Modern treatment for nasopharyngeal carcinoma: current status and prospects. Curr Opin Oncol 23: 254-258, 2011.

4. Danesh-Meyer HV: Radiation-induced optic neuropathy. J Clin Neurosci 15: 95-100, 2008.

5. Miller NR: Radiation-induced optic neuropathy: still no treatment. Clin Experiment Ophthalmol 32: 233-235, 2004.

6. Mayo C, Martel MK, Marks LB, Flickinger J, Nam J and Kirkpatrick J: Radiation dose-volume effects of optic nerves and chiasm. Int J Radiat Oncol Biol Phys 76: 28-35, 2010.

7. Demizu Y, Murakami M, Miyawaki D, et al: Analysis of vision loss caused by radiation-induced optic neuropathy after particle therapy for head-and-neck and skull-base tumors adjacent to optic nerves. Int J Radiat Oncol Biol Phys 75: 1487-1492, 2009.

8. Bhandare N, Monroe AT, Morris CG, Bhatti MT and Mendenhall WM: Does altered fractionation influence the risk of radiation-induced optic neuropathy? Int J Radiat Oncol Biol Phys 62: 1070-1077, 2005

9. Parsons JT, Bova FJ, Fitzgerald CR, Mendenhall WM and Million RR: Radiation optic neuropathy after megavoltage external-beam irradiation: analysis of time-dose factors. Int J Radiat Oncol Biol Phys 30: 755-763, 1994. 
10. Lessell S: Friendly fire: neurogenic visual loss from radiation therapy. J Neuroophthalmol 24: 243-250, 2004.

11. Arnold AC, Hepler RS, Lieber M and Alexander JM: Hyperbaric oxygen therapy for nonarteritic anterior ischemic optic neuropathy. Am J Ophthalmol 122: 535-541, 1996.

12. Levy RL and Miller NR: Hyperbaric oxygen therapy for radiation-induced optic neuropathy. Ann Acad Med Singapore 35: 151-157, 2006.

13. Chahal HS, Lam A and Khaderi SK: Is pentoxifylline plus vitamin $\mathrm{E}$ an effective treatment for radiation-induced optic neuropathy? J Neuroophthalmol 33: 91-93, 2013.

14. Farooq O, Lincoff NS, Saikali N, Prasad D, Miletich RS and Mechtler LL: Novel treatment for radiation optic neuropathy with intravenous bevacizumab. J Neuroophthalmol 32: 321-324, 2012.

15. Lee MS and Borruat FX: Should patients with radiation-induced optic neuropathy receive any treatment? J Neuroophthalmol 31: 83-88, 2011.

16. Leber KA, Bergloff J and Pendl G: Dose-response tolerance of the visual pathways and cranial nerves of the cavernous sinus to stereotactic radiosurgery J Neurosurg 88: 43-50, 1998.

17. Entezari M, Ramezani A, Pakravan M, Mahmoodi A, Hassanpour $\mathrm{H}$ and Yaseri M: Anterior ischemic optic neuropathy. Ophthalmology 119: 879-880, 2012.
18. Griffin JD and Garnick MB: Eye toxicity of cancer chemotherapy: a review of the literature. Cancer 48: 1539-1549, 1981

19. Norton SW and Stockman JR: Unilateral optic neuropathy following vincristine chemotherapy. J Pediatr Ophthalmol Strabismus 16: 190-193, 1979.

20. Di Chiro G, Oldfield E, Wright DC, et al: Cerebral necrosis after radiotherapy and/or intraarterial chemotherapy for brain tumors: PET and neuropathologic studies. AJR Am J Roentgenol 150: 189-197, 1988.

21. Hasegawa A, Mizoe JE, Mizota A and Tsujii H: Outcomes of visual acuity in carbon ion radiotherapy: Analysis of dose-volume histograms and prognostic factors. Int J Radiat Oncol Biol Phys 64: 396-401, 2006

22. Esassolak M, Karagoz U, Yalman D, Kose S, Anacak Y and Haydaroglu A: Evaluation of the effects of radiotherapy to the chiasm and optic nerve by visual psychophysical and electrophysiologic tests in nasopharyngeal carcinoma. Int J Radiat Oncol Biol Phys 58: 1141-1146, 2004. 